\title{
Analysis of self-heating of thermally assisted spin-transfer torque magnetic random access memory
}

\author{
Austin Deschenes*, Sadid Muneer, Mustafa Akbulut, Ali Gokirmak and Helena Silva
}

\author{
Full Research Paper \\ Address: \\ Electrical Engineering, University of Connecticut, Storrs, Connecticut, \\ 06082, USA \\ Email: \\ Austin Deschenes* - austin.deschenes@uconn.edu \\ * Corresponding author \\ Keywords: \\ FEM modeling; Joule heating; self-heating; spin torque transfer \\ magnetic random access memory (STT-MRAM); thermoelectrics
}

Beilstein J. Nanotechnol. 2016, 7, 1676-1683.

doi:10.3762/bjnano.7.160

Received: 11 May 2016

Accepted: 20 October 2016

Published: 11 November 2016

Associate Editor: J. Frommer

(C) 2016 Deschenes et al.; licensee Beilstein-Institut.

License and terms: see end of document.

\begin{abstract}
Thermal assistance has been shown to significantly reduce the required operation power for spin torque transfer magnetic random access memory (STT-MRAM). Proposed heating methods include modified material stack compositions that result in increased self-heating or external heat sources. In this work we analyze the self-heating process of a standard perpendicular magnetic anisotropy STT-MRAM device through numerical simulations in order to understand the relative contributions of Joule, thermoelectric Peltier and Thomson, and tunneling junction heating. A 2D rotationally symmetric numerical model is used to solve the coupled electro-thermal equations including thermoelectric effects and heat absorbed or released at the tunneling junction. We compare self-heating for different common passivation materials, positive and negative electrical current polarity, and different device thermal anchoring and boundaries resistance configurations. The variations considered are found to result in significant differences in maximum temperatures reached. Average increases of $3 \mathrm{~K}, 10 \mathrm{~K}$, and $100 \mathrm{~K}$ for different passivation materials, positive and negative polarity, and different thermal anchoring configurations, respectively, are observed. The highest temperatures, up to $424 \mathrm{~K}$, are obtained for silicon dioxide as the passivation material, positive polarity, and low thermal anchoring with thermal boundary resistance configurations. Interestingly it is also found that due to the tunneling heat, Peltier effect, device geometry, and numerous interfacial layers around the magnetic tunnel junction (MTJ), most of the heat is dissipated on the lower potential side of the magnetic junction. This asymmetry in heating, which has also been observed experimentally, is important as thermally assisted switching requires heating of the free layer specifically and this will be significantly different for the two polarity operations, set and reset.
\end{abstract}

\section{Introduction}

Spin torque transfer magnetic random access memory (STTMRAM), a type of non-volatile memory, functions through the resistance ratio between the parallel $(\mathrm{ON})$ and anti-parallel
(OFF) states of two ferromagnetic domains on either side of a thin insulating barrier. These ferromagnetic domains and the insulating barrier make up the magnetic tunnel junction (MTJ) 
of the STT-MRAM device. The device is switched between the $\mathrm{ON}$ and OFF states by passing a current of the appropriate direction through the MTJ allowing the rotational spin of tunneling carriers to "torque" the free magnetic domain into the desired alignment. STT-MRAM exhibits desirable endurance, speed, and scaling properties. The write process of STT-MRAM has been shown to be highly reactive to thermal processes [1-3] $\mathrm{Bi}$ et al. showed that utilizing external heat sources to elevate the temperature of the device by $50 \mathrm{~K}$ could reduce power consumption by up to $4.8 \%$ [2]. Bandiera et al. showed practical operation of STT-MRAM devices below the $22 \mathrm{~nm}$ node by enhanced self-heating, up to temperatures of $360 \mathrm{~K}$ to $523 \mathrm{~K}$, through manipulation of the material stack [3]. However, changing the material stack compromises the desired anisotropy levels and external heat sources impact device density. Thus, these methods for thermal assistance can be considered limited in their potential.

In this work we perform an electro-thermal analysis of the selfheating process for a standard perpendicular magnetic anisotropy STT-MRAM device (Figure 1) to understand the relative contributions of the different heat mechanisms involved and the effect of external device parameters such as passivation material, current polarity and contact configurations, and to determine how such design choices may enhance thermal assistance for STT-MRAM.

\section{Computational details Model}

We have modeled the electro-thermal behavior of the device using the "COMSOL Multiphysics Finite-Element Modeling" software. Voltage, current, and temperature are obtained by solving the coupled current (Equation 1) and heat (Equation 2) equations:

$$
\vec{J}=\sigma \vec{E}+\vec{J}_{\mathrm{e}}
$$

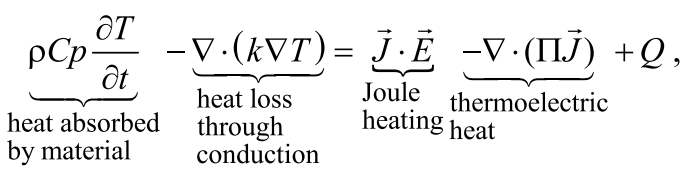

where $\vec{J}$ is the current density, $\sigma$ is the electrical conductivity, $\vec{E}$ is the electric field, $\vec{J}_{\mathrm{e}}$ is the external current source used to model the tunnel junction, $\rho$ is the mass density, $C_{p}$ is the heat capacity, $T$ is the temperature, $t$ is the time, $k$ is the thermal conductivity, and $\Pi$ is the net Peltier coefficient. Thermoelectric Peltier, and Thomson heat terms are included in the heat equation (Equation 2). Tunneling through the device is modeled using an external circuit that circumvents the thin insulating barrier with the resistance being characterized by experimental

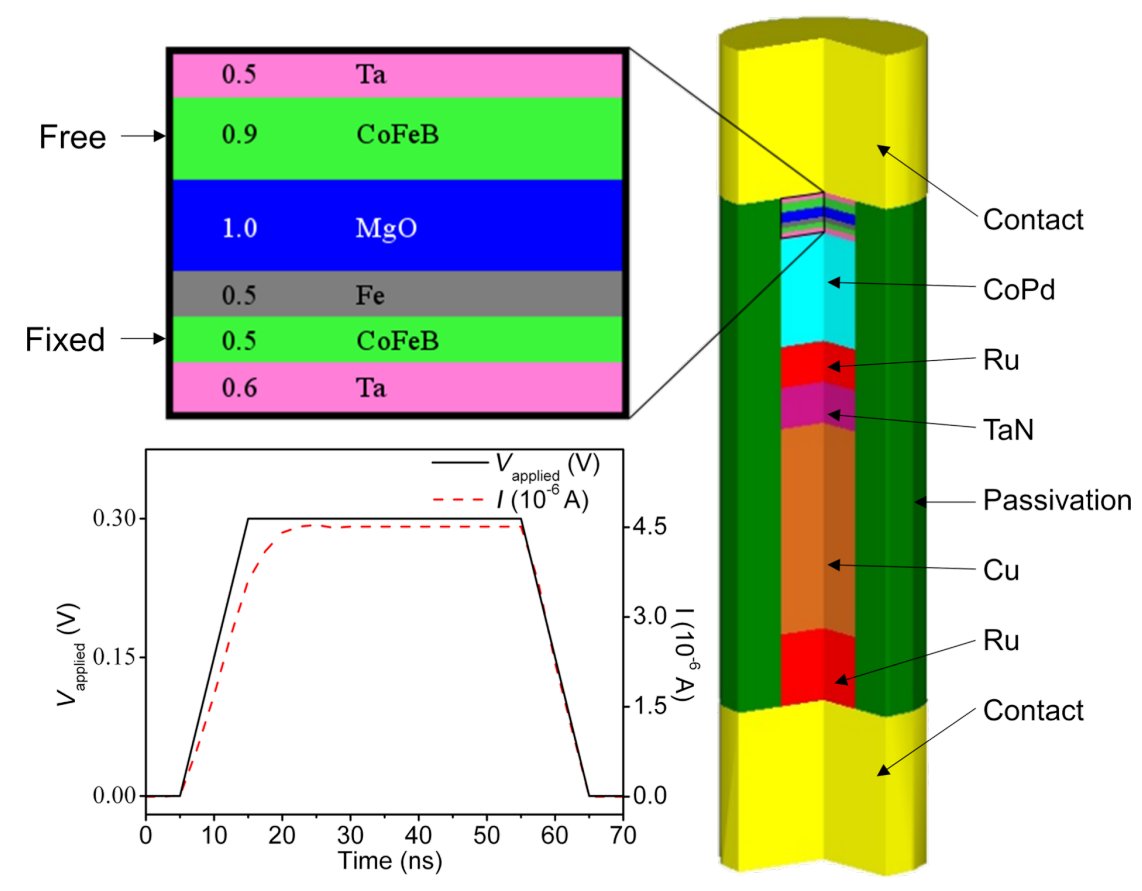

Figure 1: MRAM device structure with a stack radius of $10 \mathrm{~nm}$. Materials, with exception of contact regions, are proportional to actual device model. The CoFeB-MgO-Fe-CoFeB MTJ is shown in the enlarged image, where the dimensions are given in nanometers. The inset shows the applied voltage and resultant current waveform for $0.3 \mathrm{~V}$ pulse. 
$J-V$ curves obtained for the same junction from literature [4-7] (Figure 2).

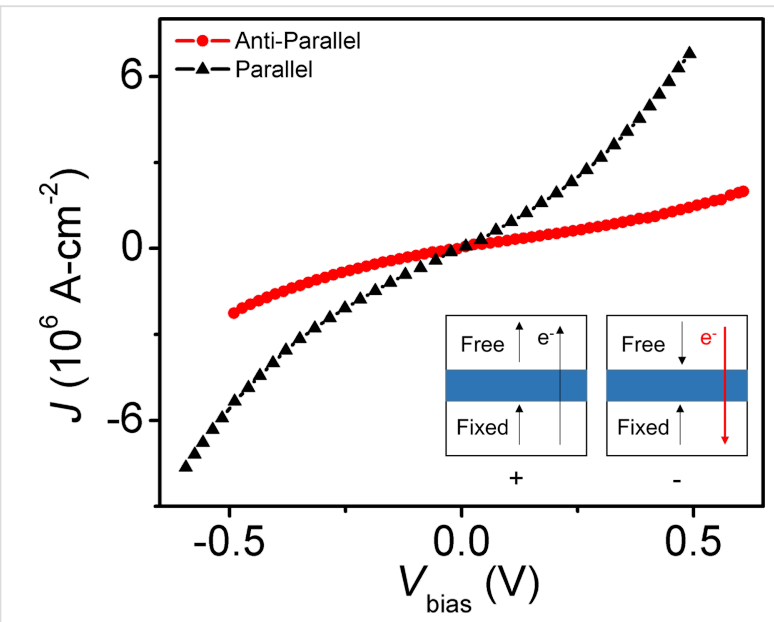

Figure 2: Characteristic $J-V$ curve built from literature [4-7] with similar $\mathrm{CoFeB} / \mathrm{MgO} / \mathrm{CoFeB}$ MTJ with thickness of the order of a few nanometers. Probes are used to find bias voltage across the tunnel junction. Curves are extrapolated via nearest-function method for $0.6 \mathrm{~V}$ simulations. Insets show the flow of carriers, and implied material properties, for a given initial free and fixed magnetic layer alignment. Simulations with initially parallel (ON) free/fixed layers use parallel properties, whereas simulations with initially anti-parallel (OFF) free/fixed layers use anti-parallel properties.

This external circuit to model the tunnel junction is the only contribution of $\vec{J}_{\mathrm{e}}$ and requires the corresponding heat contributions to be added separately as external heat sources. Heat released or absorbed at the tunneling junction $\left(Q_{t}\right)$ is modeled using the probabilistic equation for hot tunneling carrier relaxation [1]:

$$
Q_{\mathrm{t}}=\frac{\left|\vec{J}_{\mathrm{t}}\right| V_{\mathrm{t}}}{\lambda} e^{-\frac{x}{\lambda}}
$$

where $\left|\vec{J}_{t}\right|$ is the magnitude of tunneling current density, $V_{t}$ is the electric potential drop across the junction, $\lambda$ is the inelastic scattering mean free path, and $x$ is the stack position. The Peltier heat associated with the tunneling electrons is modeled using:

$$
Q_{\text {Peltier }}=\left|\vec{J}_{t}\right|\left(S_{1} T_{1}-S_{2} T_{2}\right),
$$

where $\left|\vec{J}_{\mathrm{t}}\right|$ is the tunneling current density, and $S$ and $T$ are the Peltier coefficients ( $S$ is the Seebeck coefficient, $T$ is the temperature) on either side of the junction. The heat contributions from Equation 3 and Equation 4 are released or absorbed on the appropriate side of the tunneling barrier depending on the current polarity.
The MRAM device modeled (Figure 1) is composed of the following layers: contact $|0.5 \mathrm{Ta}| 0.9 \mathrm{CoFeB}$ (free) $|1 \mathrm{MgO}|$ $0.5 \mathrm{Fe} \mid 0.5 \mathrm{CoFeB}$ (fixed) | 0.6 Ta $|[0.25 \mathrm{Pd} \mid 0.8 \mathrm{Co}] \times 10|$ $4 \mathrm{Ru}|4 \mathrm{TaN}| 20 \mathrm{Cu}|7 \mathrm{Ru}|$ contact, where the numbers before the alloy composition of each layer represent the thickness of that layer in nanometers. The radius of the cylindrical material stack is $10 \mathrm{~nm}$. The passivation layer surrounding the stack is $5 \mathrm{~nm}$ thick. In the material stack the CoFeB layers are the ferromagnetic domains of the $\mathrm{MTJ}, \mathrm{MgO}$ is the thin insulating layer, and $\mathrm{Ta} / \mathrm{CoPd} / \mathrm{Ru}$ is the synthetic antiferromagnetic layer. The material stack modeled is from a device reported by Worledge et al. [8]. The thermal stability factor of the free ferromagnetic layer of the device, derived from Equation 5 using materials properties from [8,9], is $38.7 k_{\mathrm{B}} \mathrm{T}$ :

$$
\Delta_{\text {perp }}=\frac{M_{\mathrm{s}} \cdot\left(H_{\mathrm{k}}^{\text {perp }}-4 \pi M_{\mathrm{s}}\right) \cdot V}{2 k_{\mathrm{B}} T},
$$

where $\Delta_{\text {perp }}$ is the perpendicular thermal stability factor, $M_{\mathrm{s}}$ is the saturation magnetization, $H_{\mathrm{k}}^{\text {perp }}$ is the perpendicular effective anisotropy field, $V$ is the volume of the free layer, $k_{\mathrm{B}}$ is the Boltzmann constant, and $T$ is the local temperature.

Temperature-dependent materials properties [7,10-14] (Figure 3) are used for $\mathrm{CoFeB}, \mathrm{MgO}$, and $\mathrm{Fe}$. The temperaturedependent thermal conductivity of $\mathrm{CoFeB}$ is calculated using the Wiedemann-Franz law:

$$
k=L \sigma T
$$

where $L$ is the Lorenz number. It is assumed the annealing temperature was sufficiently low so as to not cause crystallization [7] on the $\mathrm{MgO}-\mathrm{CoFeB}$ interface, leaving the $\mathrm{CoFeB}$ completely amorphous. The $\mathrm{Pd}-\mathrm{Co}$ layers are treated as an alloy due to the large number of sub-nanometer thick layers. The temperature-dependent materials properties of the PdCo alloy were derived using the behavior of similar alloys [10] transposed onto point data [15]. The material properties of the various common passivation materials considered [16] (Table 1) are from the element library of COMSOL for $\mathrm{SiO}_{2}$ and $\mathrm{Si}_{3} \mathrm{~N}_{4}$, and from literature for low-temperature plasma-enhanced chemical vapor deposition $\mathrm{SiO}_{2}$ [2,17] and $\mathrm{Si}_{3} \mathrm{~N}_{4}$ [18]. The "ON" and "OFF" states of the device are represented via interchangeable sets of materials properties for the CoFeB layers $[4,13]$ (Figure 2, Figure 3) that are chosen based on the desired switching operation to be simulated (anti-parallel properties to simulate an OFF-ON switching operation, parallel properties for the converse operation, see inset in Figure 2). 


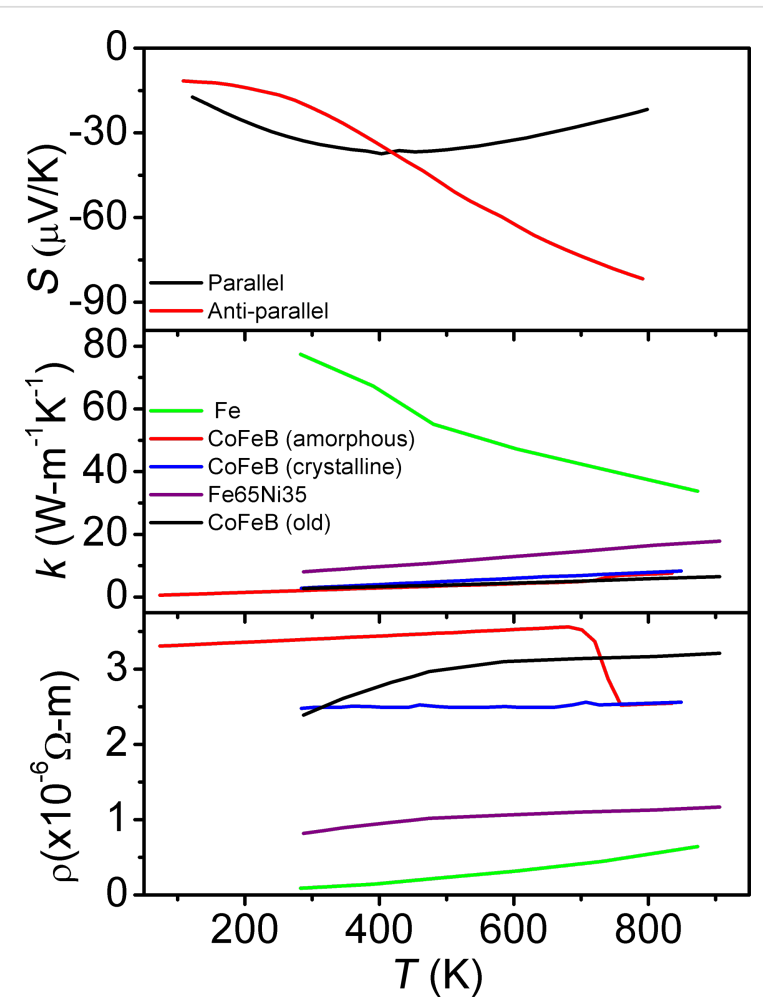

Figure 3: Graph of materials properties used in model construction. From top to bottom: Seebeck values of CoFeB for parallel (ON) and anti-parallel (OFF) states [11], thermal conductivity of materials [8,10], electrical resistivity of materials [8]. Crystalline and amorphous CoFeB values came from literature [12].

\begin{tabular}{|llll}
\hline \multicolumn{6}{l}{ Table 1: Properties of passivation materials. } \\
material & $\begin{array}{l}\text { relative } \\
\text { permittivity }\end{array}$ & $\begin{array}{l}\text { thermal conductivity } \\
(\mathrm{W} / \mathrm{m} \cdot \mathrm{K})\end{array}$ & $\begin{array}{l}\text { heat capacity } \\
(\mathrm{J} / \mathrm{kg} \cdot \mathrm{K})\end{array}$ \\
\hline $\mathrm{Si}_{3} \mathrm{~N}_{4}{ }^{* \mathrm{~b}}$ & 7 & 30 & 170 \\
$\mathrm{Si}_{3} \mathrm{~N}_{4}$ & 7.5 & 30 & 700 \\
$\mathrm{SiO}_{2}{ }^{*}$ & 5 & 1.1 & 650 \\
$\mathrm{SiO}_{2}$ & 3.9 & 1.4 & 730 \\
\hline
\end{tabular}

aproperties at room temperature are given. Values are from the "COMSOL" element library for $\mathrm{SiO}_{2}$ and $\mathrm{Si}_{3} \mathrm{~N}_{4}$, (high-temperature growth or deposition, considered here as a limit case) and from literature for $\mathrm{SiO}_{2}{ }^{*}[1,15]$ and $\mathrm{Si}_{3} \mathrm{~N}_{4}{ }^{*}[16]$. ${ }^{\text {b The asterisk indicates material }}$ properties for low-temperature plasma-enhanced chemical vapor deposition (PECVD) silicon dioxide or silicon nitride.

\section{Simulation procedures}

Analysis of the self-heating of the device is performed for different passivation materials (Table 1), positive and negative current polarity, and four contact configurations (Figure 4), using voltage pulses as shown in the inset of Figure 1. The bias voltage dependence of the TMR is not considered and the resistance values of the MTJ in the ON and OFF states, derived from Figure 2, are assumed to be constant during the switching pulses. A positive voltage is applied at point $\mathrm{A}$ and ground at point $\mathrm{B}$ in Figure 4 (positive polarity, to simulate $\mathrm{ON}-\mathrm{OFF}$ switching) or the converse (negative polarity, to simulate OFF-ON switching). The thermal boundary conditions, represented as $\mathrm{C}$ in Figure 4, are set to $300 \mathrm{~K}$ at all times. The peak temperatures within the free $\mathrm{CoFeB}$ layer, fixed $\mathrm{CoFeB}$ layer, and for the whole device are recorded for each pulse as $T_{\text {free}}$, $T_{\text {fixed }}$, and $T_{\text {peak }}$, respectively.

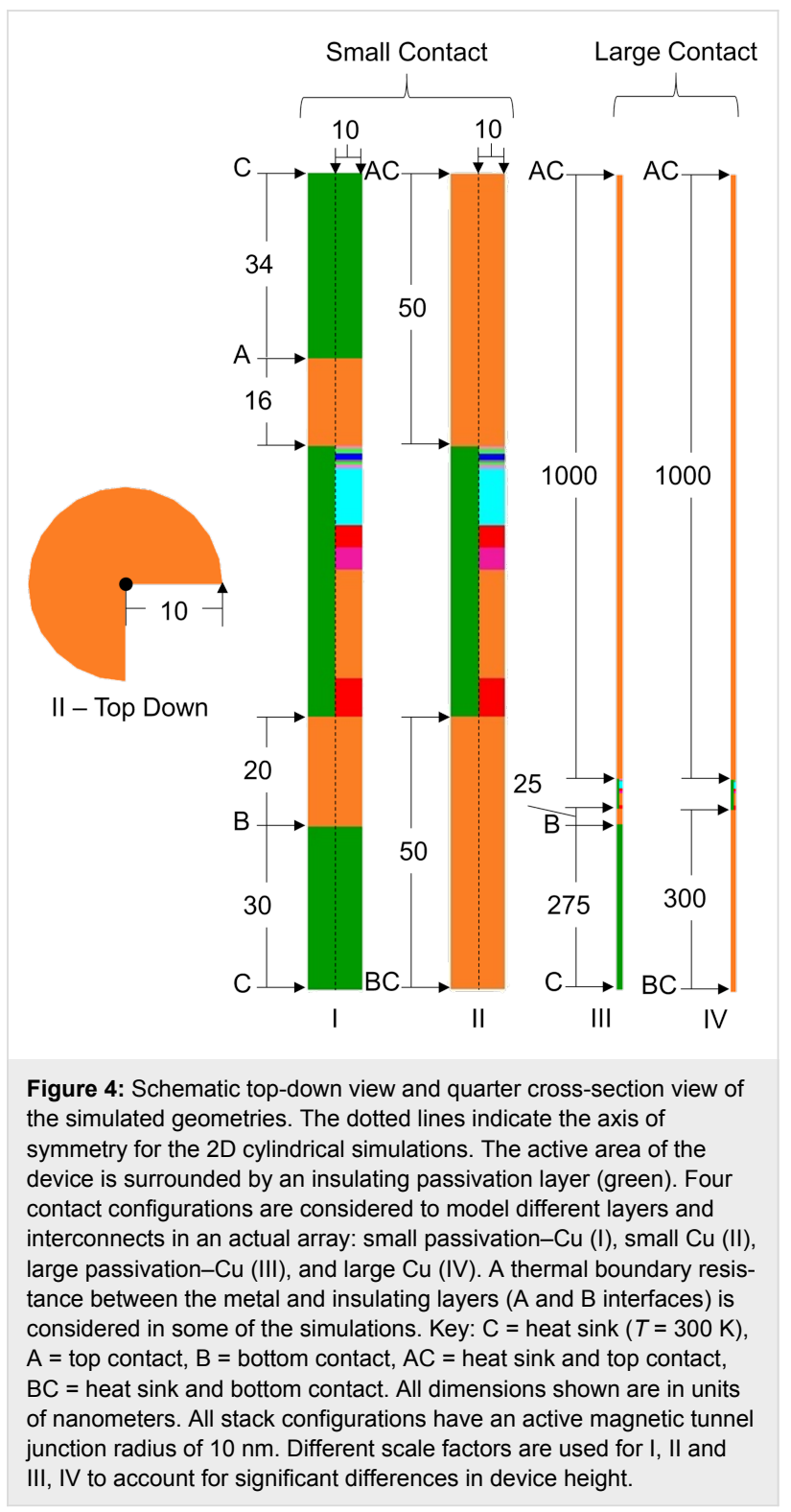

The contact regions in Figure 1 are changed between sets of simulations in order to model the device in different thermal anchoring conditions. The four configurations used to model these contact regions are shown in Figure 4. To model a discrete STT-MRAM cell, the configurations I and II are simulated and referred to as "small contact" devices. To model a device in a 
memory array, the "large contact" configurations III and IV are used to emulate large thermal coupling [19]. To model different heat paths through metal and passivation layers, the configurations with passivation- $\mathrm{Cu}$ contacts (I, III) are simulated with and without thermal boundary resistances (TBR) applied on the passivation- $\mathrm{Cu}$ interfaces. This TBR is modeled as a $1 \mathrm{~nm}$ thick virtual layer with a thermal conductivity of $0.041 \mathrm{~W} / \mathrm{m} \cdot \mathrm{K}$.

Simulation sets are performed for configurations II and IV in positive polarity, with pulse amplitudes ranging from 0.2 to $0.4 \mathrm{~V}$, with all passivation materials considered in Table 1. Additional simulations are run for $\mathrm{SiO}_{2}$ passivation layer, which exhibits the most favorable contributions to self-heating (see Results and Conclusion), on configurations I to IV with pulse amplitude ranging from 0.2 to $0.6 \mathrm{~V}$ for both positive and negative polarity to further analyze the effects of thermal anchoring and current polarity.

\section{Results and Discussion}

Asymmetry of heating around the MTJ, shown by the simulation results in Figures 5-8, is a result of the asymmetric heat contributions from $Q_{\text {Peltier }}$ and $Q_{\text {Tunneling }}$ and interfacial layers with high thermal resistance surrounding the thin insulating $\mathrm{MgO}$ layer. The asymmetry in heating increases with overall heating as shown below in Figure 7 and Figure 8 for configurations II and IV. Experimental observations of asymmetric heating in MRAM devices have been reported earlier by

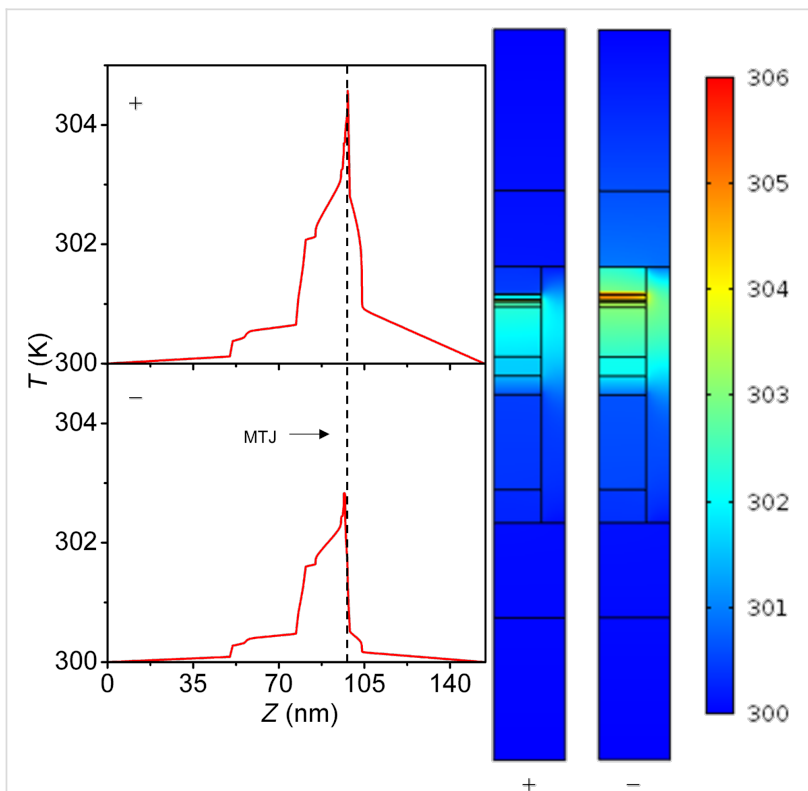

Figure 5: Thermal profiles of configuration II with $\mathrm{SiO}_{2}$ passivation with an applied voltage of $0.3 \mathrm{~V}$ in "positive" polarity (top) and "negative" polarity (bottom). Left figures show the temperature as a function of the vertical position with $0 \mathrm{~nm}$ being the bottom of the device. The dotted line in the plots represents the center of the MTJ. Note the asymmetry around the MTJ.

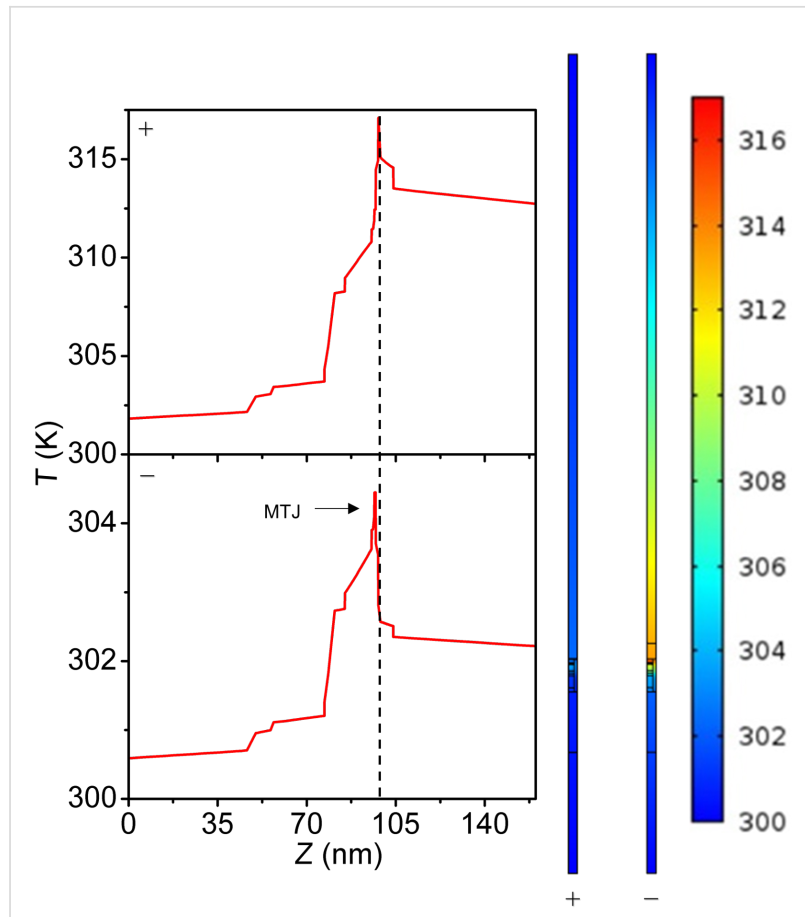

Figure 6: Thermal profiles of configuration $\mathrm{IV}$ with $\mathrm{SiO}_{2}$ passivation with an applied voltage of $0.3 \mathrm{~V}$ in "positive" polarity (top) and "negative" polarity (bottom). The left panels show the temperature as a function of the vertical position. The dotted line in the plots represents the center of the MTJ. Note the asymmetry around the MTJ. The free layer in the magnetic tunnel junction reaches ca. $316 \mathrm{~K}$ in positive polarity while it only reaches ca. $303 \mathrm{~K}$ in negative polarity.

Gapihan et al. who predicted that the favorable current direction can result in a $10 \%$ reduction in the heating power density required for switching [20].

The temperature $T_{\text {peak }}$ of the small-contact configurations at expected operational voltages of STT-MRAM $(0.3 \mathrm{~V})$ range from $305 \mathrm{~K}$ for configuration II in positive polarity with $\mathrm{Si}_{3} \mathrm{~N}_{4}$ passivation, to $424 \mathrm{~K}$ for configuration I with TBR in positive polarity with $\mathrm{SiO}_{2}$ passivation.

Large-contact configurations exhibit higher asymmetric tendencies and, on average, have a higher $T_{\text {peak }}$ by $11.8 \mathrm{~K}$ compared to observations for similar small-contact simulations (same passivation material, voltage applied, and polarity). This is the result of more pronounced geometric asymmetry and a larger heat capacity of the configurations III and IV. At typical operational voltages of STT-MRAM large-contact configurations have $T_{\text {peak }}$ ranging from $312 \mathrm{~K}$ for configuration IV in positive polarity with $\mathrm{Si}_{3} \mathrm{~N}_{4}$ passivation to $326 \mathrm{~K}$ for configuration III in positive polarity with $\mathrm{SiO}_{2}$ passivation.

$\mathrm{SiO}_{2}$ passivation provides superior amplification to the selfheating of STT-MRAM with $T_{\text {peak }}$ that are, on average, $2.6 \mathrm{~K}$ 
higher than comparable simulations for the other considered passivation materials (low-temperature PECVD $\mathrm{SiO}_{2}$ and $\mathrm{Si}_{3} \mathrm{~N}_{4}$, and $\mathrm{Si}_{3} \mathrm{~N}_{4}$ ). This is observable in Figure 7 and Figure 8 .

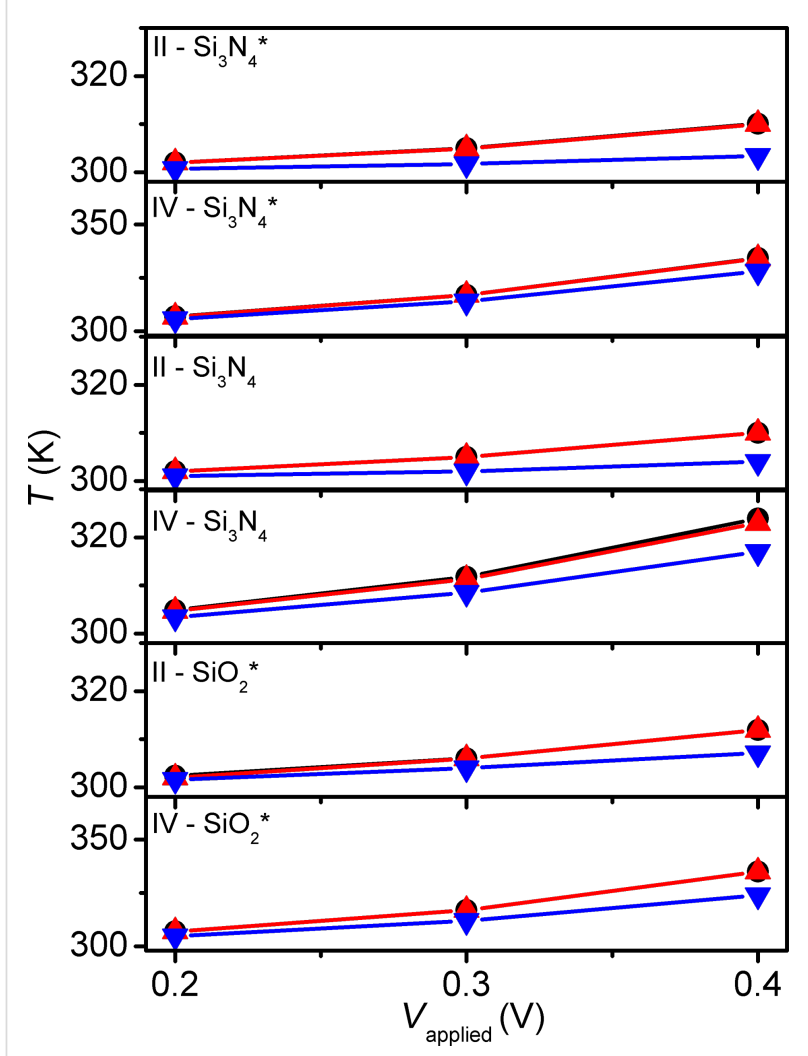

Figure 7: Results for different passivation materials. $T_{\text {peak }}$ (black circles), $T_{\text {free }}$ (red upward triangles) and $T_{\text {fixed }}$ (blue downward triangles), recorded for configurations II and IV from 0.2 to $0.4 \mathrm{~V}$ in positive polarity.

For the extended analysis of $\mathrm{SiO}_{2}$ passivation it is observed that small-contact configurations attain peak temperatures, on average, 111.2 K higher than their large-contact counterparts (i.e., the temperatures of configurations I and I-TBR are higher than those of configurations III and III-TBR). This is the result of the passivation material inhibiting heat flow (which is not present in configuration II), and smaller contact regions having lower heat capacity. Additionally, positive polarity yields, on average, $7.9 \mathrm{~K}$ higher $T_{\text {peak }}$ than negative polarity. This is due to reduced current in the negative polarity switching (device is initially in off state) and reduced distance to the thermal boundary for heat generated on the bottom of the MTJ. Assuming constant resistances for the two states - which is equivalent to assuming switching occurs only at the end of the pulses - together with temperature-dependent changes of electrical and thermal conductivity may result in a small underestimation or overestimation of the difference in heating between the positive and negative polarities. Nevertheless, the simulation results appear

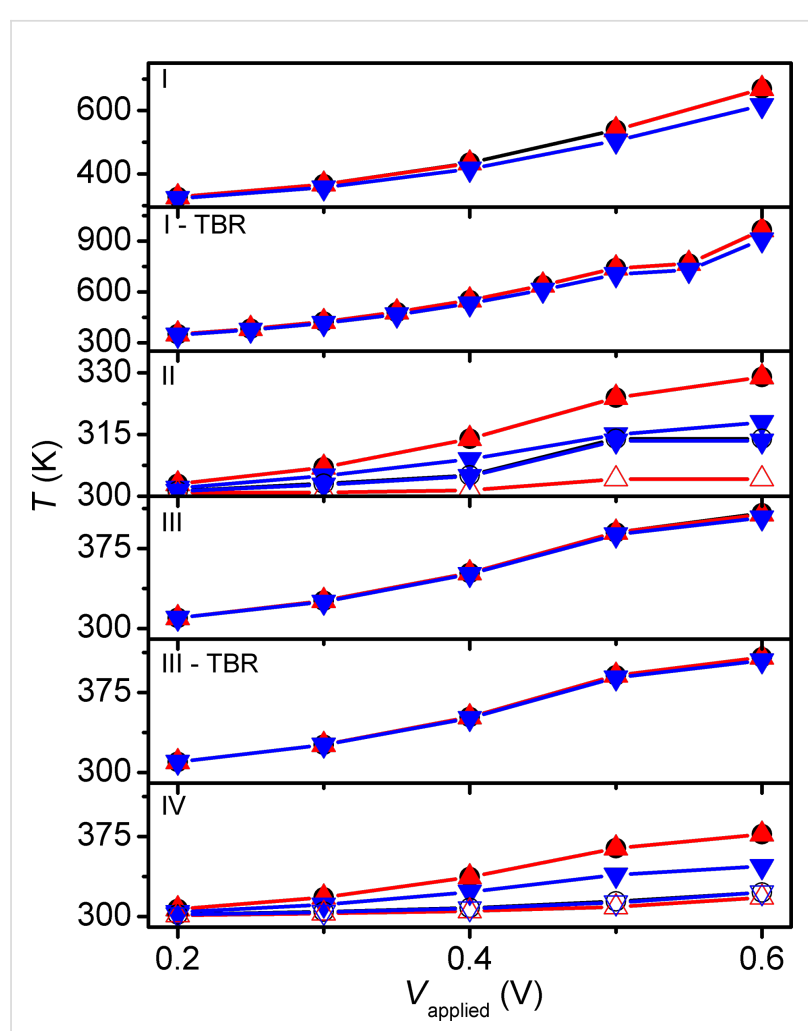

Figure 8: $T_{\text {peak }}$ (black circles), $T_{\text {free }}$ (red upward triangle) and $T_{\text {fixed }}$ (blue downward triangles), recorded for large- and small-contact configurations. Simulations carried out for $\mathrm{SiO}_{2}$ passivation. Configurations II and IV feature positive polarity (solid symbols) and negative polarity (open symbols). Configurations I and III are simulated with and without thermal boundary resistance (TBR) at the passivation-Cu interfaces.

to be in reasonable agreement with the experimental observations of a reduction by ca. $10 \%$ in required power density for the positive polarity reported by Gapihan et al. [20]. Expected temperature asymmetries for opposite current flow under the same resistance state should yield higher temperatures on the lower potential side of the MTJ, similar to the patterns observed in Figures 5-8. However, these cases are not simulated as the thermal effect is mainly of interest for current flow direction and resistance state combinations that result in device switching. Partial inclusion of the different self-heating mechanisms in the simulations shows the relative importance of each (Figure 9). The largest contributor to device heating, by a significant margin, is $Q_{\mathrm{t}}$. The Peltier and Joule heating in the device are minimal in comparison. As a result, heating is largely localized in the MTJ region as seen in Figure 5 and Figure 6.

Simulations were performed to observe the dependence of thermal behavior on the thickness of the passivation layer and no significant changes were observed between 5 and $20 \mathrm{~nm}$ thick layers (except at high voltages and low thermal anchoring where heat saturation occurs). 


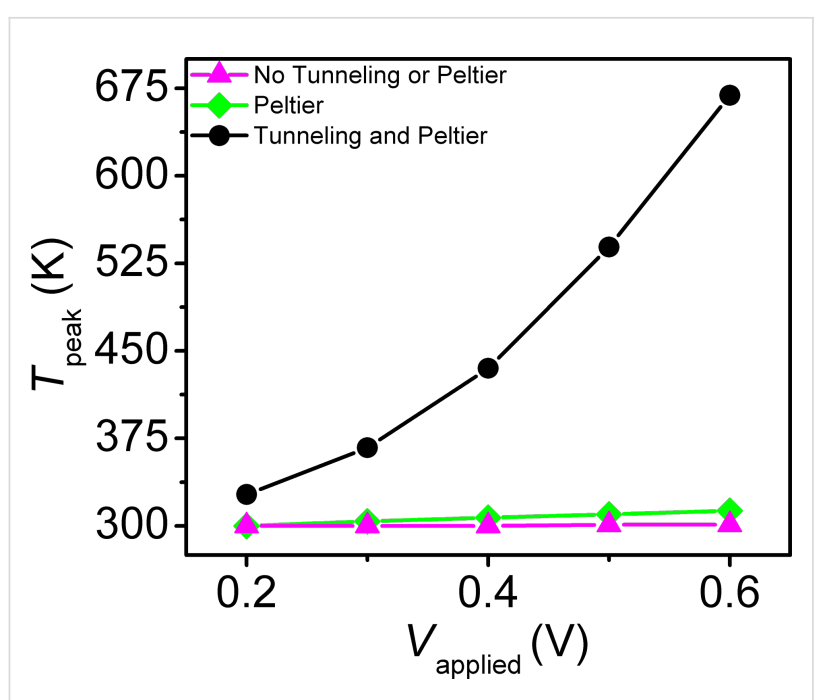

Figure 9: Individual heat contributions from Peltier effect and tunneling around the MTJ for configuration I with $\mathrm{SiO}_{2}$ passivation material.

\section{Conclusion}

In this work, we analyzed the electro-thermal behavior of a standard STT-MRAM device for different passivation layers, current polarity and contact configurations and found that selfheating varies significantly and that, for some cases, it can effectively produce required temperatures of ca. $350 \mathrm{~K}$ [2] to about $523 \mathrm{~K} \mathrm{[3]} \mathrm{for} \mathrm{thermal} \mathrm{assistance} \mathrm{at} \mathrm{expected} \mathrm{operation}$ voltages. The use of high-temperature $\mathrm{PECVD} \mathrm{SiO}_{2}$ (with slightly higher thermal conductivity and heat capacity compared to low-temperature PECVD $\mathrm{SiO}_{2}$ ) as the passivation material results in significantly higher self-heating compared to the other common passivation materials $\left(\mathrm{Si}_{3} \mathrm{~N}_{4}\right.$ and low-temperature PECVD $\mathrm{Si}_{3} \mathrm{~N}_{4}$ and $\mathrm{SiO}_{2}$ ). Positive polarity leads to a $10 \mathrm{~K}$ average temperature increase at negative polarity. Interestingly, the results also show that due to tunneling heat, Peltier effect, device geometry, and numerous interfacial layers around the MTJ, the heating on the lower potential side of the junction for a given polarity is higher. This asymmetry is important because thermal assistance requires heating of the free ferromagnetic domain, causing thermal destabilization. But the free domain only experiences significant heating when the device is in positive polarity. Thus care must be taken to ensure the free domain achieves a sufficiently high temperature independent of the overall device temperature.

\section{Acknowledgements}

The authors are thankful to Daniel Worledge for valuable discussions and suggestions that led to this work. A. Deschenes was supported by an REU supplement made to NSF Grant ECCS 1150960. S. Muneer, A. Gokirmak and H. Silva were partially supported by the U.S. Department of Energy through Basic Energy Sciences Award DE-SC005038. M. Akbulut was partially supported by the U.S. National Science Foundation through Award ECCE 1150960. The authors would also like to acknowledge Nadim Kanan, Nicholas Williams, and Zachary Woods for general discussions and helpful criticism.

\section{References}

1. Prejbeanu, I. L.; Kerekes, M.; Sousa, R. C.; Sibuet, H.; Redon, O.; Dieny, B.; Nozières, J. P. J. Phys.: Condens. Matter 2007, 19, 165218. doi:10.1088/0953-8984/19/16/165218

2. Bi, X.; Li, H.; Kim, J.-J. Analysis and Optimization of Thermal Effect on STT-RAM Based 3-D Stacked Cache Design. In 2012 IEEE Computer Society Annual Symposium on VLSI (ISVLSI), Aug 19-21, 2012; IEEE Computer Society: Piscataway, NJ, U.S.A., 2012; pp 374-379. doi:10.1109/ISVLSI.2012.56

3. Bandiera, S.; Sousa, R. C.; Marins de Castro, M.; Ducruet, C.; Portemont, C.; Auffret, S.; Vila, L.; Prejbeanu, I. L.; Rodmacq, B.; Dieny, B. Appl. Phys. Lett. 2011, 99, 202507. doi:10.1063/1.3662971

4. Dankert, A.; Dulal, R. S.; Dash, S. J. Sci. Rep. 2013, 3, 3196. doi:10.1038/srep03196

5. Chen, E.; Apalkov, D.; Diao, Z.; Driskill-Smith, A.; Druist, D.; Lottis, D.; Nikitin, V.; Tang, X.; Watts, S.; Wang, S.; Wolf, S. A.; Ghosh, A. W.; Lu, J. W.; Poon, S. J.; Stan, M.; Butler, W. H.; Gupta, S.; Mewes, C. K. A.; Mewes, T.; Visscher, P. B. IEEE Trans. Magn. 2010, 46, 1873-1878. doi:10.1109/TMAG.2010.2042041

6. Zeng, Z. M.; Khalili Amiri, P.; Rowlands, G.; Zhao, H.; Krivorotov, I. N.; Wang, J.-P.; Katine, J. A.; Langer, J.; Galatsis, K.; Wang, K. L.; Jiang, H. W. Appl. Phys. Lett. 2011, 98, 072512. doi:10.1063/1.3556615

7. Bakker, F. L.; Slachter, A.; Adam, J.-P.; van Wees, B. J. Phys. Rev. Lett. 2010, 105, 136601. doi:10.1103/PhysRevLett.105.136601

8. Worledge, D. C.; Hu, G.; Abraham, D. W.; Sun, J. Z.; Trouilloud, P. L.; Nowak, J.; Brown, S.; Gaidis, M. C.; O’Sullivan, E. J.; Robertazzi, R. P. Appl. Phys. Lett. 2011, 98, 022501. doi:10.1063/1.3536482

9. Liu, X.; Zhang, W.; Carter, M. J.; Xiao, G. J. Appl. Phys. 2011, 110, 033910. doi:10.1063/1.3615961

10. Ho, C. Y.; Ackerman, M. W.; Wu, K. Y.; Havill, T. N.; Bogaard, R. H.; Matula, R. A.; Oh, S. G.; James, H. M. J. Phys. Chem. Ref. Data 1983, 12, 183-322. doi:10.1063/1.555684

11. Fulkerson, W.; Moore, J. P.; McElroy, D. L. J. Appl. Phys. 1966, 37, 2639. doi:10.1063/1.1782098

12. Kumar, G. S.; Prasad, G.; Pohl, R. O. J. Mater. Sci. 1993, 28 , 4261-4272. doi:10.1007/BF01154931

13. Walter, M.; Walowski, J.; Zbarsky, V.; Münzenberg, M.; Schäfers, M.; Ebke, D.; Reiss, G.; Thomas, A.; Peretzki, P.; Seibt, M.; Moodera, J. S.; Czerner, M.; Bachmann, M.; Heiliger, C. Nat. Mater. 2011, 10, 742-746. doi:10.1038/nmat3076

14. Kalinin, Yu. E.; Sitnikov, A. V.; Stognei, O. V.; Zolotukhin, I. V.; Neretin, P. V. Mater. Sci. Eng., A 2001, 304-306, 941-945. doi:10.1016/S0921-5093(00)01606-3

15. Loram, J. W.; Williams, G.; Swallow, G. A. Phys. Rev. B 1971, 3 , 3060-3065. doi:10.1103/PhysRevB.3.3060

16. Lipkin, L. A.; Palmour, J. W. IEEE Trans. Electron Devices 1999, 46, 525-532. doi:10.1109/16.748872

17. Idris, I.; Sugiura, O. Jpn. J. Appl. Phys., Part 1 1998, 37, 6562-6568. doi:10.1143/JJAP.37.6562

18. Piccirillo, A.; Gobbi, A. L. J. Electrochem. Soc. 1990, 137, 3910. doi:10.1149/1.2086326 
19. Kent, A. D.; Worledge, D. C. Nat. Nanotechnol. 2015, 10, 187-191. doi:10.1038/nnano.2015.24

20. Gapihan, E.; Hérault, J.; Sousa, R. C.; Dahmane, Y.; Dieny, B.; Vila, L.; Prejbeanu, I. L.; Ducruet, C.; Portemont, C.; Mackay, K.; Nozières, J. P. Appl. Phys. Lett. 2012, 100, 202410. doi:10.1063/1.4719663

\section{License and Terms}

This is an Open Access article under the terms of the Creative Commons Attribution License

(http://creativecommons.org/licenses/by/4.0), which permits unrestricted use, distribution, and reproduction in any medium, provided the original work is properly cited.

The license is subject to the Beilstein Journal of

Nanotechnology terms and conditions:

(http://www.beilstein-journals.org/bjnano)

The definitive version of this article is the electronic one which can be found at: doi:10.3762/bjnano.7.160 